\title{
Survival probability of an edge Majorana in a 1-D p-wave superconducting chain under sudden quenching of parameters
}

\author{
Atanu Rajak ${ }^{1}$ and Amit Dutta ${ }^{2}$ \\ ${ }^{1}$ CMP Division, Saha Institute of Nuclear Physics, \\ 1/AF Bidhannagar, Kolkata 700 064, India and \\ ${ }^{2}$ Department of Physics, Indian Institute of Technology Kanpur, Kanpur 208016, India
}

\begin{abstract}
We consider the temporal evolution of a zero energy edge Majorana of a spinless $p$-wave superconducting chain following a sudden change of a parameter of the Hamiltonian. Starting from one of the topological phases that has an edge Majorana, the system is suddenly driven to the other topological phase or to the (topologically) trivial phases and also to the quantum critical points (QCPs) separating these phases. The survival probability of the initial edge Majorana as a function of time is studied following the quench. Interestingly when the chain is quenched to the QCP, we find a nearly perfect oscillations of the survival probability, indicating that the Majorana travels back and forth between two ends, with a time period that scales with the system size. We also generalize to the situation when there is a next-nearest-neighbor hopping in superconducting chain and there resulting in a pair of edge Majorana at the each end of the chain in the topological phase. We show that the frequency of oscillation of the survival probability gets doubled in this case. We also perform an instantaneous quenching the Hamiltonian (with two Majorana modes at each end of the chain) to an another Hamiltonian which has only one Majorana mode in equilibrium; the MSP shows oscillations as a function of time with a noticeable decay in the amplitude. On the other hand for a quenching which is reverse to the previous one, the MSP decays rapidly and stays close to zero with fluctuations in amplitude.
\end{abstract}

PACS numbers: 74.40.Kb,74.40.Gh,75.10.Pq

\section{INTRODUCTION}

The topological properties of a $p$-wave spin-less superconductor, introduced by Kitaev $\underline{\underline{1}}$ has been studied from different points of views in recent years ${ }^{2}-\underline{\underline{z}}$. (For a review $\left.\sec ^{\frac{9}{}}\right)$. A spinless $p$-wave superconductor is a topological system which has edge Majorana modes as midgap excitations that are guaranteed by particle-hole symmetry of the system. The superconducting pairing term of such a Hamiltonian actually induces the zero energy excitations in the system. The distinct phases of this system are characterized by the presence (or absence) of the zero energy Majorana modes at the ends of a long and open chain. The phases those have zero energy Majorana modes are called topologically non-trivial and others are topologically trivial. The number of these modes are topological invariant; this value does not change until the system crosses the phase boundary between the topological and the trivial phases as happens in the case of a topological insulator $\underline{10,11}$. It has been proposed that the proximity effect between the surface states of a strong topological insulator and $s$-wave superconductor can generate a two dimensional state strongly resembling a $p$ wave superconductor which can hosts Majorana states at the vortices 12 .

Recent experiments have also been able to detect the signature of these Majorana modes in the zero-bias transport properties of nanowires coupled to superconductors ${ }^{13}-16$. Additionally, it has also been demonstrated experimentally that these Majorana modes can be hybridized and be made to appear or disappear by tuning the chemical potential of a similar system across a topo- logical phase transition 17 . There are also some discrepancies in experimental results with the theoretical predictions of Majorana fermions which has been discussed in Ref. 18. We note that there are several recent studies on the possible decoherence of a Majorana qubit 19,20 and the possibility of dynamical generation of Majorana Fermions ${ }^{7.21}$; the sudden quench of a 1-d Majorana chain has also been studied through its topological signature in the entanglement spectrum ${ }^{22}$.

In parallel, there are a plethora of recent studies of the non-equilibrium dynamics of quantum many body systems driven across quantum critical points $23-25$. The failure of achieving a perfectly adiabatic evolution close to a quantum critical point $(\mathrm{QCP})$, where the relaxation time is very large, produce excitations in the system which leads to non-zero defect density in the final state that the system reaches following the quenching. This defect density scales with the rate of quenching which is usually determined by the Kibble-Zurek (KZ) scaling relation $26-28$ given in terms of the rate of quenching and some of the critical exponents associated with the QCP across which the system is driven. On the other hand, for a sudden quench across a QCP, the scaling of the defect density generated in the final state is given in terms of the magnitude of the change of the quenched parameter and the critical exponents 29,30 . Recently, the studies of the connection between quenching dynamics and the topological order ${ }^{31}-33$ and also the dynamics of an edge state existing in the topological phase ${ }^{34}$ have attracted the attention of the scientists. It has been established that following a slow quenching across a QCP, an edge Majorana of 1-D $p$-wave superconductor shows different scaling from that 
predicted by the Kibble-Zurek scaling indicating that the quenching dynamics of an edge state is non-universal and depends on the topology of the system 35 . In a similar spirit, the dynamics of an edge state of a topological insulator in a ribbon geometry has been explored after the system is suddenly quenched to the QCP (between the topological and trivial insulator phases) and the signature of a surviving spin-Hall current that oscillates in time has been observed ${ }^{36}$. We note that very recently the possibility of thermalization of non-local topological order has been studied by tracking the time evolution of the string correlations in a spin-1 chain in its Haldane phase following a sudden quench ${ }^{37}$.

In this paper, we consider a spinless $p$-wave superconducting chain of finite but long system size and study the dynamics of a zero energy edge Majorana after suddenly quenching the system from one topologically non-trivial phase to another (or to the topologically trivial phase) and also to the QCP separating these phases. Following the quench, the edge Majorana gets coupled to the bulk modes and hence is expected to decohere. We address the question whether this happens for all quenching schemes. Analyzing the Majorana survival probability (MSP), we here demonstrate that there could be situations when the edge Majorana survives and oscillates between two edges of the chain.

The paper is organized in the following manner: in the next section (Sec II) we discuss the model Hamiltonian discussing the equilibrium phase diagram with different topological and non-topological phases. In Sec. III we shall define the quantity which we call the MSP and measure this after a rapid quench of a parameter of the superconducting chain. In subsequent subsections, we consider different paths for quenching in the phase diagram and present our numerical results. We extend the results to 1-D chain to study of $p$-wave superconductor with next nearest neighbor hopping in the Sec. IV We also calculate the MSP in Sec. $\mathrm{V}$ when the system is quenched between a phase with two Majorana modes to a phase with one Majorana mode. Finally, our results are summarized in Sec. VI.

\section{MODEL}

The Hamiltonian of a one-dimensional $p$-wave superconducting system of spinless (or spin polarized) fermions with system size $N$ is given by

$$
\begin{aligned}
H & =\sum_{j=1}^{N}\left[-w\left(a_{j}^{\dagger} a_{j+1}+a_{j+1}^{\dagger} a_{j}\right)+\Delta\left(a_{j} a_{j+1}+a_{j+1}^{\dagger} a_{j}^{\dagger}\right)\right] \\
& -\sum_{j=1}^{N} \mu\left(a_{j}^{\dagger} a_{j}-1 / 2\right)
\end{aligned}
$$

where $w, \Delta$ and $\mu$ denote nearest-neighbor hopping strength, superconducting gap and on-site chemical potential, respectively. We have considered spacing of the lattice as unity and also Planck constant, $\hbar=1$ throughout the paper. The annihilation and creation operators $a_{j}\left(a_{j}^{\dagger}\right)$ obey the usual anticommutation relations $\left\{a_{j}, a_{l}\right\}=0$ and $\left\{a_{j}, a_{l}^{\dagger}\right\}=\delta_{j l}$. The periodic boundary condition of the lattice $\left(a_{N+1}=a_{1}\right)$ makes the Hamiltonian in Eq. (1) translationally invariant and it can be then diagonalized in momentum basis, $a_{k}=\frac{1}{\sqrt{N}} \sum_{j=1}^{N} a_{j} e^{-i k j}$. This leads to a particle-hole symmetric dispersion given by

$$
E_{k}= \pm 2 w \sqrt{(\eta+\cos k)^{2}+\xi^{2} \sin ^{2} k},
$$

where we have introduced two relative parameters $\xi=$ $\Delta / w$ and $\eta=\mu / 2 w$. The bulk energy gap $\left(2 E_{k}\right)$ vanishes at certain values of $\xi$ and $\eta$ for some specific $k$ modes. The phase diagram of the model with three distinct phases (denoted by I, II and III) is shown in Fig. 1 One observes that $\eta= \pm 1$ are two quantum critical lines with critical modes $k_{c}=\pi$ and 0 (for which the energy gap vanishes), respectively, whereas for the critical line $\xi=0$ (with $\eta$ lies between -1 and 1 ) with $k_{c}=\cos ^{-1}(-\eta)$.

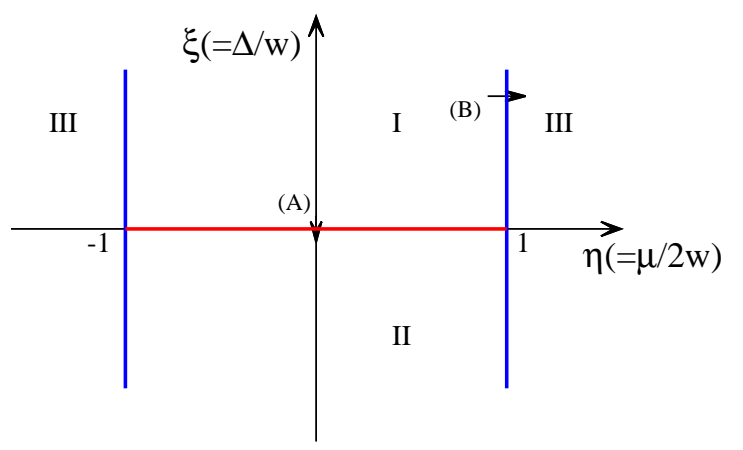

FIG. 1. (Color online) Phase diagram of the 1D $p$ - wave superconducting system (see Eq.(1)). Phases $I$ and $I I$ are topologically non-trivial while phase III is topologically trivial. Quenching paths A and B are shown in the phase diagram.

We can represent the Hamiltonian (Eq.(10) in terms of Majorana fermion operators

$$
c_{2 j-1}=a_{j}+a_{j}^{\dagger} \quad c_{2 j}=\frac{1}{i}\left(a_{j}-a_{j}^{\dagger}\right)
$$

These Majorana operators are Hermitian and satisfy the relations $c_{2 j-1}^{2}=c_{2 j}^{2}=1$ and $\left\{c_{j}, c_{l}\right\}=2 \delta_{j l}$; these imply that to one fermionic site $j$ there is two Majorana sites or equivalently, a Majorana fermion can viewed as occupying half of a state. Then the Eq. (1) with an open 
boundary conditions can be re-written as

$$
\begin{aligned}
H & =\frac{i}{2} \sum_{j=1}^{N-1}\left[(-w+\Delta) c_{2 j-1} c_{2 j+2}+(w+\Delta) c_{2 j} c_{2 j+1}\right] \\
& -\frac{i}{2} \sum_{j=1}^{N} \mu c_{2 j-1} c_{2 j} .
\end{aligned}
$$

The zero energy modes of the Hamiltonian in Eq.(4) distinguish different phases (I, II and III) in the phase diagram (see Fig. 1). Phase $\mathrm{I}(\xi>0$ and $|\eta|<1)$ is one of the topological non-trivial phases, due to the presence of two isolated Majorana modes at the two edges of a open and long chain (see Fig. (2). It can be shown using a special condition i.e, for $\Delta=w$ and $\mu=0$, when, as can be seen from Eq.(4) that the Majorana operators $c_{1}$ and $c_{2 N}$ do not appear in the Hamiltonian leading to two unpaired zero energy Majorana fermions $c_{1}$ and $c_{2 N}$ at the left and right end of the chain, respectively. Similarly, for the phase II $(\xi<0$ and $|\eta|<1)$ two isolated Majorana modes, $c_{2}$ and $c_{2 N-1}$, exist at the two ends of the chain. As a result, the ground state of the system is twofold degenerate with definite fermionic parity for this two phases. (One can combine the two unpaired Majorana fermions to get a usual complex fermion, giving two degenerate ground states with fermionic occupation number 0 or 1.) We note that the phases I and II are different with respect to the nature of edge modes in two ends of the chain. While Phase I hosts odd (even) site edge Majorana modes at the left (right) of the open and long chain, it is the other way round in Phase II. The phase III $(|\eta|>1)$ is topologically trivial with no edge Majorana modes. This can be illustrated considering the special limit $\Delta=w=0, \mu \neq 0$, when all the Majorana fermions are connected pairwise for each fermionic lattice site and consequently there is no isolated edge states. The system has a topological invariant (TI) through which we can distinguish different phases; this is known as the winding number ${ }^{38.39}$ which takes values $-1,1$ and 0 in phases I, II and III, respectively. One can also transform the Hamiltonian (Eq.(44) to a spin-1/2 XY model Hamiltonian with a transverse field by using the Jordan-Wigner transformation ${ }^{40}$.

\section{QUENCH DYNAMICS AND RESULTS}

In this section, we will study the survival probability of an edge Majorana when the superconducting chain is quenched from one phase to the other or to the QCP separating two phases as shown in Fig. 1. Immediately after the quench the quantum state is not an the eigenstate of the final Hamiltonian and hence evolves in time with time evolution dictated by the final Hamiltonian.

To illustrate the method to be employed here, we represent the Eq4in a generic quadratic form with Majorana operators

$$
H=\frac{i}{4} \sum_{j, k=1}^{2 N} A_{j k} c_{j} c_{k},
$$

where $A$ is a real skew-symmetric $2 N \times 2 N$ matrix and the $c_{j}$ are the Majorana operators; the eigenvalues of $A$ appear in pairs as $\pm i \epsilon_{j}(j=1,2, \ldots, 2 N)$. The zero eigenvalues of the above Hamiltonian will be even in number and these are called zero modes. For an open and long chain the wave functions of zero energy modes can be made real and they have finite gap with respect to other eigenvalues. These properties lead to identification of the end modes as Majorana states ${ }^{7}$. Two isolated edge Majorana states existing in the phase I is shown in Fig. 2. these are obtained by diagonalizing the Hamiltonian in Eq.(5) with given parameter values to obtain the zero eigenvalues and constructing the appropriate real wave functions.

Let us now analyze the energy spectrums for the Majorana chain for open and and periodic boundary conditions as shown in Fig. [3 for $\xi \in[-1,1]$ with $\eta=0$. We have set here $w=1$; a convention that would be followed for rest of the paper. We can clearly identify that the Hamiltonian in Eq.(5) carries two zero energy modes in both the phases I and II for a open chain; but periodic chain has no zero energy states and hence it is a purely non-topological system.

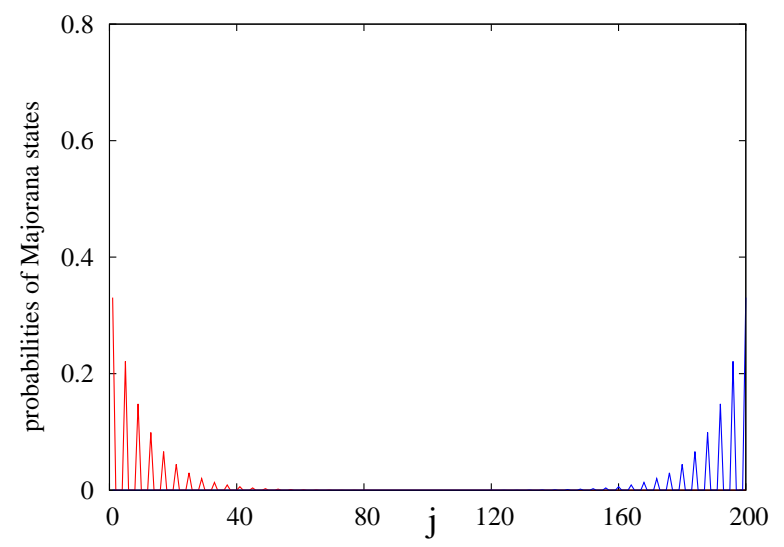

FIG. 2. (Color online) Two isolated Majorana states are localized in two edges of a 100-site open Majorana chain in phase I ( $\xi=0.1$ and $\eta=0.0) . \quad j$ labels as Majorana sites $1,2, \ldots 200$. One can see that here probability is non-zero only if $j$ is odd (even) for the left (right) end of Majorana chain.

We now quench rapidly a parameter of the Hamiltonian in Eq. (15) considering different paths on the phase diagram (Fig. 1) at the instant $t=0$ and study the time evolution of a zero energy Majorana mode. Following a sudden quench the time evolved state of the Majorana at 

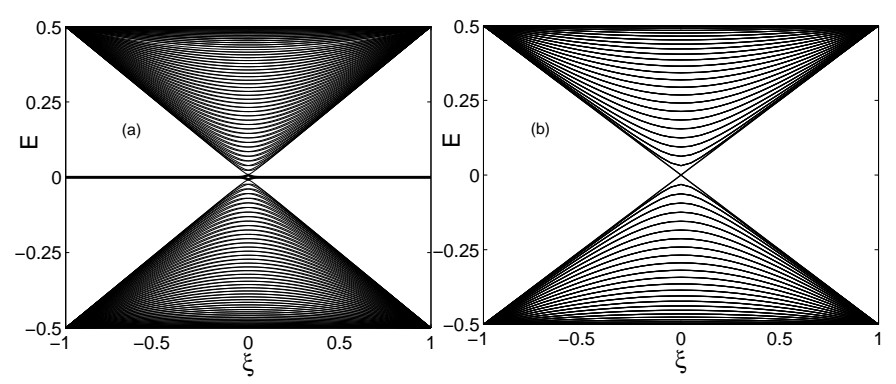

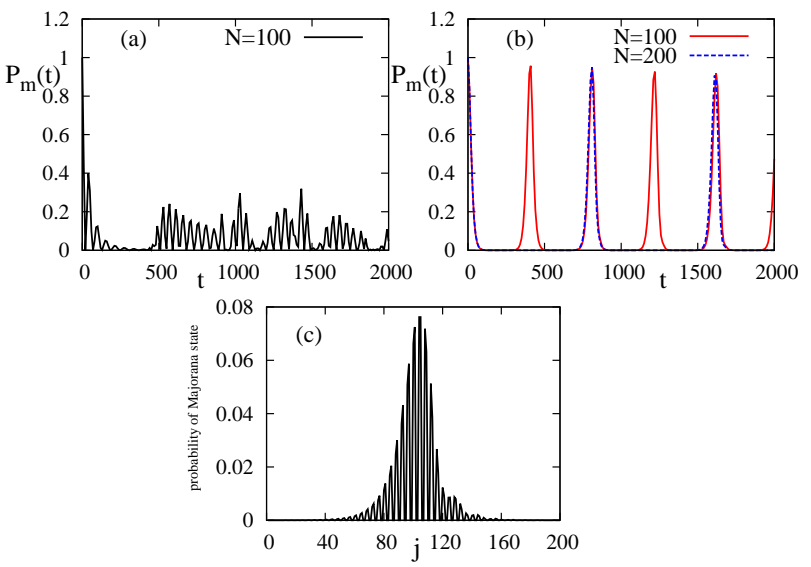

FIG. 3. Energy spectrum of the Majorana chain of system size $N=100$ as a function of $\xi$ with $w=1$ and $\eta=0$ using (a) open boundary condition (OBC) and (b) periodic boundary condition (PBC), respectively. Note that two zeroenergy Majorana modes are present in case (a) but not in case (b). We mention that the energy is scaled by a factor $1 / 4$ in Eq.(5) in comparison to Eq.(2).

time $t$ can be written as

$\left|\psi_{m}(\xi, \eta, t)\right\rangle=\sum_{n=1}^{2 N} e^{-i E_{n} t}\left|\Phi_{n}\left(\xi^{\prime}, \eta^{\prime}\right)\right\rangle\left\langle\Phi_{n}\left(\xi^{\prime}, \eta^{\prime}\right) \mid \psi_{m}(\xi, \eta)\right\rangle$,

where $\left|\psi_{m}(\xi, \eta)\right\rangle$ is an initial end Majorana state for the parameters $\xi$ and $\eta$, and $\left|\Phi_{n}\left(\xi^{\prime}, \eta^{\prime}\right)\right\rangle$ are the eigenstates of the final Hamiltonian with new parameters $\xi^{\prime}, \eta^{\prime}$ while $E_{n}$ 's are the eigenvalues of the final Hamiltonian. These are obtained by diagonalizing the Hamiltonian in Eq. (5) numerically for the initial and final parameter values. To study the dynamics of the zero energy Majorana mode after a sudden quench, we now define the Majorana survival probability (MSP) $P_{m}(t)$ (which is infact the Loschmidt echo (LE) $\stackrel{41,42}{ }$ ) defined as

$$
P_{m}(t)=\left.\left.\left|\sum_{n=1}^{2 N}\right|\left\langle\psi_{m}(\xi, \eta) \mid \Phi_{n}\left(\xi^{\prime}, \eta^{\prime}\right)\right\rangle\right|^{2} e^{-i E_{n} t}\right|^{2}
$$

which we shall analyze below for different quenching paths.

\section{A. Quenching along different paths}

We have considered a path A $(\eta=0)$ in the Fig. 1. Along this path we change the parameter $\xi$ suddenly from phase I to II.The probability defined in Eq.(7), decays rapidly with time and remains minimum having some noisy fluctuation of small amplitude (Fig. 4(a)) for first quench scheme (phase I to II). This signifies that the Majorana mode de-coheres with time, but not completely due to unitary evolution of the system.

Let us now focus on the most interesting situation which emerges when we quench the chain to the quantum critical point $(\mathrm{QCP})(\xi=0, \eta=0)$; we find that
FIG. 4. (Color online) Majorana survival probability of a zero A and probability of Majorana after quench. (a) MSP decays rapidly and does not revive significantly for quenching from phase I $(\xi=0.1)$ to phase II $(\xi=-0.1)$. (b) Quenching from phase I $(\xi=0.1)$ to the $\mathrm{QCP}(\xi=0.0)$, MSP shows nearly perfect collapse and revival with time $t$ and scales linearly with the system size $N$. (c) Probability of the end Majorana at time $t=100$ after quench at the QCP with the Majorana site $j$. This shows that at this instant the probability of Majorana is maximum around the center of the chain.
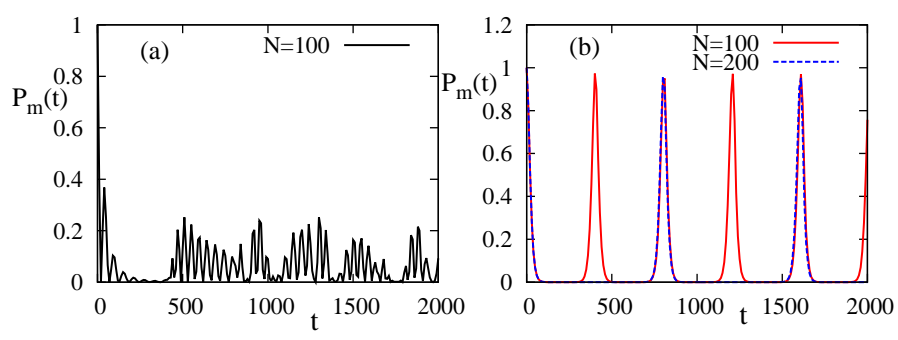

FIG. 5. (Color online) The probablities of an end mode $(\xi=$ 1.0 and $\eta=0.9)$ after a quench (a) at a point $(\xi=1.0$ and $\eta=1.1)$ of phase III and (b) at a QCP $(\xi=1.0$ and $\eta=1.0)$ along the path $\mathrm{B}$ show same behavior as of the path $\mathrm{A}$.

the MSP of an edge Majorana mode shows collapse and revival with time (Fig. $4(b)$ ). This can be attributed to the nearly equi-spaced levels of the bulk close at $\xi=0$ (see Fig. [3); moreover there is bulk bandgap $E_{g} \sim 1 / N$ as dynamical critical exponent associated with this QCP is unity.

The significant contribution to the summation of Eq. (7) arises because of the overlap of the edge Majorana state with of these (nearly equispaced) low-energy states of the bulk. Then the Eq. (7) represents Fourier series of a periodic function with fundamental frequency as an energy difference of two consecutive equispaced levenergy Majorana for different quench schemes along the path 
els i.e., proportional to $1 / N$; this in turn implies that the quasi period of revival should scale with the chain length $N$. One can confirm this prediction from Fig. 4(b) where the time period of oscillation getting double when system size becomes twice of earlier. These observations lead to the conclusion that when the chain quenched at the QCP separating two topological phases the edge Majorana mode of Fig. 2 oscillates between two ends of the chain (see Fig. 4(c)) with the time period of this oscillation being proportional to the size of the spin chain. However, the amplitude of revival decreases with time due to the coupling of the Majorana with bulk levels of higher energy as it oscillates back and forth between the two edges.

To analyze this oscillation of the Majoranas further, we refer to the situation with the periodic boundary condition; although in this case, though the edge $\mathrm{Ma}-$ jorana does not exist but the structure of bulk energy levels remains the same. We can then write the Hamiltonian in Eq. (1) as the direct product of decoupled $2 \times 2$ matrices in each Fourier mode $k$; the bulk energy dispersion is given by the Eq.(2); at the critical point $(\xi=0, \eta=0)$, the bulk energy gap vanishes for the critical mode $k_{c}=\pi / 2$. Now, if we expand $E_{k}$ close to the critical mode $k_{c}=\pi / 2$ the first non-vanishing term in $E_{k}$ is linear order in $k$ and second term will be proportional to $k^{3}$ (where we have rescaled $\left(k-k_{c}\right) \rightarrow k$ ). If one starts from the ground state in one of the phases which evolves with the critical Hamiltonian, the modulus of the overlap between the initial state and the time evolved state (or the Loschmidt echo) takes a form: $\mathcal{L}(t)=\prod_{k>0}\left(1-A_{k} \sin ^{2}\left(E_{k} t\right)\right) \stackrel{41-43}{\underline{n}}$, where $A_{k}$ is a sufficiently slowly varying function of $k$ and each term has a periodicity $\pi / E_{k}$. We now focus on the modes close to $k \rightarrow 0$ separated by $\Delta k=2 \pi / N$; these modes interfere constructively at time instants given $\Delta E_{k} t=p \pi$, where $p$ is an integer; yielding revival time for these modes given by $t_{k}=\frac{1}{2} p N\left|\partial E_{k} / \partial k\right|$. For a strictly linear dispersion (valid for modes with $k \rightarrow 0$ ), when $\left|\partial E_{k} / \partial k\right|$ is independent of $k$, many modes add up constructively and one finds a pronounced revival of the overlap at intervals which scale with chain length $N$. Noting that the bulk energy difference, $\Delta E=E_{n}-E_{n-1} \approx 2 \pi / N$ (with $w=1$ ), between two consecutive low-energy states close to $\xi=0$. As a consequence, the time-period of revival is given by

$$
T=\frac{2 \pi}{\Delta E} \propto N .
$$

However, the amplitude of the revival decay with time; if one considers the next higher order non-linear term in the expansion of $E_{k} \sim k^{3}$, these modes do not interfere constructively and there is an eventual decay in the amplitude as the Majorana oscillates back and forth between the edges; asymptotically the MSP saturates at a finite value for a finite size system.

Let us now choose a path $\mathrm{B}(\xi=1.0)$ that crosses or terminates at the quantum critical line separating topo- logical (I) and non-topological (III) phases. The quenching results (Figs. 5(a),(b)) for the both cases i.e., quenching from phase I to phase III and upto QCP are exactly same as of path A which can be explained using a similar set of arguments.
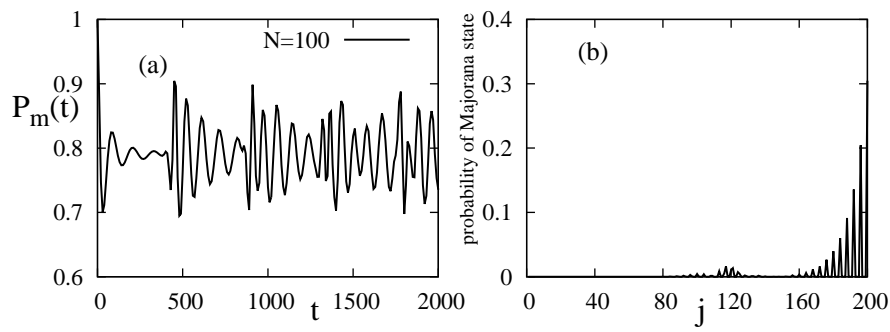

FIG. 6. System is suddenly quenched within the same phase (I) from $(\xi=0.2$ and $\eta=0.0)$ to $(\xi=0.1$ and $\eta=0.0)$ and MSP becomes (a) rapidly fluctuating function of time $t$ with a mean value of nearly 0.8 and (b) probability of the end Majorana at $t=100$, with the Majorana sites $j$ after quench.

\section{B. Quenching within the same phase}

In this case the parameters in the Hamiltonian in Eq.(4) is quenched within the same phase (phase I). Due to quenching MSP decays, but it does not collapse at zero because bulk of the system has a pronounced gap and the system does not go to the gapless QCP. Also, there is no perfect overlap due to the unequal spacing of energy levels. The MSP rapidly fluctuates haphazardly with a mean value close to unity (see Fig. 6(a)). We conclude that the zero energy Majorana mode is mainly localized at the end of the chain after this quenching (see Fig. 6(b)).

\section{QUENCHING IN THE PRESENCE OF A NEXT-NEAREST-NEIGHBOR INTERACTION}

In this section we introduce the Hamiltonian for 1-D $p$-wave superconductor with next-nearest-neighbor hopping and superconducting pairing which lead to two isolated edge Majorana modes at each end of the chain with open boundary condition. Our interest is to study how does the presence of two Majorana modes at each end shows up in the MSP following a sudden quenching. The Hamiltonian for such a 1-D chain is given by ${ }^{8}$,

$$
\begin{aligned}
H & =\sum_{j=1}^{N-1}\left[-w\left(a_{j}^{\dagger} a_{j+2}+a_{j+2}^{\dagger} a_{j}\right)+\Delta\left(a_{j} a_{j+2}+a_{j+2}^{\dagger} a_{j}^{\dagger}\right)\right] \\
& -\sum_{j=1}^{N} \mu\left(a_{j}^{\dagger} a_{j}-1 / 2\right) .
\end{aligned}
$$


In this case $w$ and $\Delta$ are the next-nearest-neighbor hopping strength and superconducting pairing term, respectively. In an identical fashion to that used for the nearest neighbor chain (see Eq.(2)), one can find out the energy spectrum for the Hamiltonian (Eq.(99)), given by

$$
E_{k}= \pm 2 w \sqrt{(\eta+\cos 2 k)^{2}+\xi^{2} \sin ^{2} 2 k} .
$$

Comparing with Eq. (2), we note that due to the next nearest neighbor interaction, there is a shift of wave vector $k \rightarrow 2 k$ in the spectrum.

To describe the topological properties of this system, we represent the Hamiltonian in Eq.(9) using Majorana fermions with an open boundary condition, which now takes the form

$$
\begin{aligned}
H & =\frac{i}{2} \sum_{j=1}^{N-1}\left[(-w+\Delta) c_{2 j-1} c_{2 j+4}+(w+\Delta) c_{2 j} c_{2 j+3}\right] \\
& -\frac{i}{2} \sum_{j=1}^{N} \mu c_{2 j-1} c_{2 j} .
\end{aligned}
$$

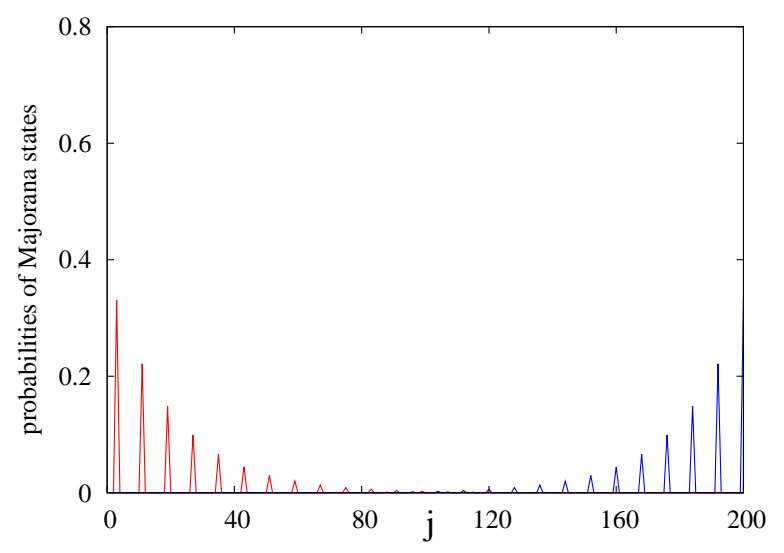

FIG. 7. (Color online) Two zero energy Majorana modes of a system Hamiltonian defined in Eq.(11) exist in two edges of a 100-site open Majorana chain in phase $\mathrm{I}(\xi=0.1$ and $\eta=0.0$ ). Similarly to Fig. 2. here also $j$ labels as Majorana sites $1,2, \ldots 200$. The probabilities are non-zero only if $j$ is odd (even) for the left (right) end of Majorana chain.

The phase diagram also consists of two topological phases (I, II) and a non-topological phase (III); however, the phases I and II have two isolated Majorana fermions in each end of the chain. We can illustrate this again using an extreme limit; for $\xi=1$ and $\eta=0$, the chain carries two zero-energy Majorana modes $c_{1}$ and $c_{3}$ in left end and also two modes $c_{2 N-2}$ and $c_{2 N}$ in right end. On the other hand, for $\xi=-1$ and $\eta=0$ lying in phase II, there are two zero-energy Majorana modes $c_{2}$ and $c_{4}$ in the left end and other two modes $c_{2 N-3}$ and $c_{2 N-1}$ in the right end of the open and long chain. In the Fig. 7 we have shown one set of equilibrium edge Majorana modes for phase I in two ends of the chain

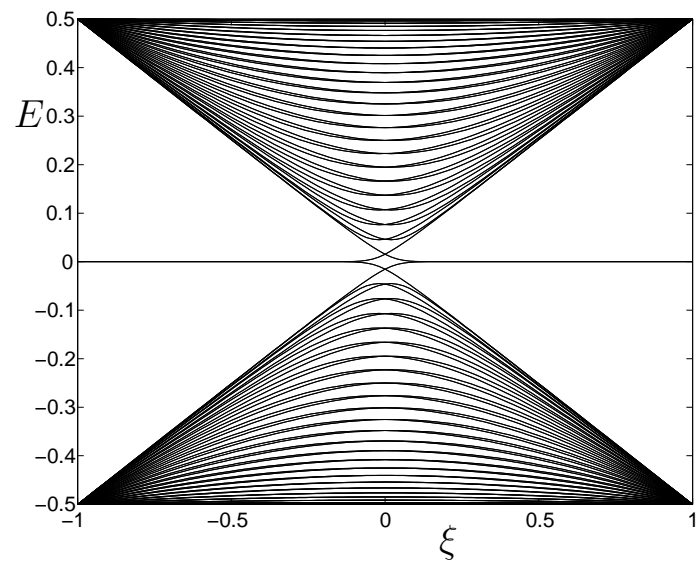

FIG. 8. Energy spectrum of the Majorana chain (with only next-nearest-neighbor hopping and interaction as defined in Eq.(9) ) as a function of $\xi$ with $\eta=0$ and $N=100$ sites for open boundary condition. In this case also a energy scale difference exists (see caption of Fig. 33).

obtained via diagonalizing the Hamiltonian (5) for the present case. We notice that here the peaks of probabilities occur in double difference in Majorana sites compare to earlier case (see Fig. 22). This can be attributed to the next-nearest-neighbor hopping and superconducting interaction. From Eq10 we can see that there are two critical lines $\eta= \pm 1$ with critical modes $k_{c}=\pi / 2$ and 0 , respectively. Another critical line, $\xi=0$ (separating phases I and II) exists with critical mode $k_{c}=\frac{1}{2} \cos ^{-1}(-\eta)$.

In this case also we have measured MSP for one of the edge Majoranas at one end following a sudden change of the parameter in the Hamiltonian (Eq. (11)) considering the same paths as in Sec. IIIA. One can see in the Fig. 9(a) as usual rapid decay of the probability (introduced in Eq.(77) with time having some noisy fluctuation of small amplitude when the system is quenched from phase I to II along the path A $(\eta=0)$. Now, we concentrate on the Fig. 9(b) which shows a noticeable change in the time period of collapse and revival of the probability in comparison to Fig. 因(b) following a quench to QCP $(\xi=0, \eta=0)$ from phase I the time period of oscillation becomes half of the earlier case (see Fig. प(b)) for equal system size. This can be explained using the bulk energy spectrum of the system. We have discussed in Sec III A that this perfect collapse and revival of MSP is a consequence of nearly equi-spaced levels of the bulk spectrum close to $\mathrm{QCP}(\xi=0, \eta=0)$. In the present case energy spacings close to QCP get doubled compared to the nearest neighbor 1-D chain for the same system size. This leads to energy difference between two consecutive equispaced levels is proportional to $2 / N$. The doubling of spacings between two consecutive energy levels close to QCP can also be seen comparing Fig. 3 to Fig. 8 . This in fact explains the reduction in the time period of collapse and revival of MSP in the present situation. These observations imply that when this system 

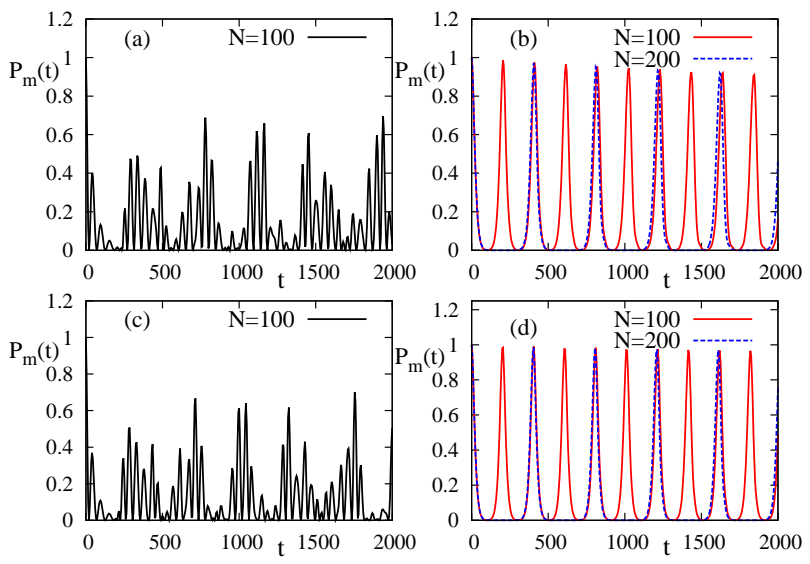

FIG. 9. (Color online) $P_{m}(t)$ of a right end zero energy Majorana mode after quenching along different paths. (a) MSP decays rapidly and stays minimum with some noisy fluctuation of small amplitude when the system (see Eq.(9)) is quenched from phase I $(\xi=0.1)$ to the phase II $(\xi=-0.1)$ along the path A. (b) For quenching to QCP $P_{m}(t)$ is nearly perfect oscillatory function of time $t$ with a interesting fact that its time period becomes half of the earlier case (see Fig. 4) and scales linearly with the system size $N$. (c) Time variation of MSP with $\xi=1.0$ and $\eta=0.9$ following a quench to a point $(\xi=1.0$ and $\eta=1.1)$ and $(\mathrm{d})$ the QCP $(\xi=1.0$ and $\eta=1.0)$ along the path $\mathrm{B}$ show same behavior as of the path $\mathrm{A}(\eta=0)$.

is quenched suddenly to QCP separating two topological phases the edge Majorana takes half of the time in comparison to nearest-neighbor case (see Fig. (4) for a full oscillation between two edges of the chain. In this case the end Majorana enters in the bulk with hopping to the next-nearest-neighboring sites and thereby avoiding subsequent sites.

We have also checked that the MSP for the same quenching schedules with the path $\mathrm{B}(\xi=1.0)$ and the results come out to be same as of path $\eta=0$ (see Figs. 9(c),(d)). We can argue similar to above to explain the doubling of the frequency in collapse and revival of MSP.

\section{QUENCHING BETWEEN A PHASE WITH TWO MAJORANA MODES TO A PHASE WITH ONE MAJORANA MODE}

In this section, we shall label the Hamiltonians in Eqs 1 and 9 as $H_{1}$ and $H_{2}$, respectively (both with open boundary conditions). Now we consider a time-dependent Hamiltonian as

$$
H(t)=\Theta(t) H_{1}+[1-\Theta(t)] H_{2}
$$

where $\Theta(t)$ is a function whose value is 0 for $t<0$ and 1 for $t>0$, which implies that at $t=0$ Hamiltonian describing the system suddenly changes from $H_{2}$ to $H_{1}$ with the hopping amplitude and the superconducting gap being identical (in the topological phase I) in both the cases while the chemical potential $\mu$ set equal to zero. We recall that in the equilibrium the Hamiltonian $H_{1}$ has two Majoranas $c_{1}$ and $c_{2 N}$ in the two ends of the chain, and similarly the Hamiltonian $H_{2}$ has a pair of Majoranas $c_{1}$ and $c_{3}$ in left and also $c_{2 N-2}$ and $c_{2 N}$ in right end.

Our purpose here is to study the survival probability of one of the edge Majoranas residing at the left end when the Hamiltonian is changed suddenly from $H_{2}$ to $H_{1}$; result is presented in Fig. [10 where we find oscillation in the survival probability. Referring to the situation depicted in Fig. 10(a), we note that the survival probability of $c_{3}$ oscillate as a function of time even though the $c_{3}$ Majorana should not exist in the final phase of the Hamiltonian in Eq. (12) and eventually the survival probability vanishes in the asymptotic limit. Remarkably, the period of oscillation is again found to scale with $N$. We have also probed the reverse situation, when the Hamiltonian is changed from $H_{1}$ to $H_{2}$, where we find a rapid decoherence of the Majorana $c_{1}$ as shown in the right panel of Fig. (10).

Remarkably, we find similar oscillations are obtained by changing $H_{2}$ to $H_{1}$ when the latter is kept at the critical point with $\xi=0$ (see Fig. 11). There is no such oscillation in the reverse case.

Oscillations obseved in the left panels of Figs. 10 and 11 imply that apparently the system behaves in such a way that it is quenched to critical point of the final Hamiltonian eventhough there is a small $\Delta$; this is a surprising and remarkable result.
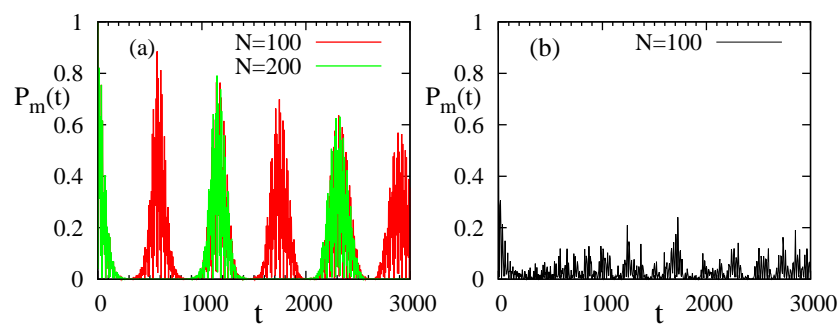

FIG. 10. System changes suddenly from a phase with two Majorana fermions to a phase which contains only one Majorana mode at each end of the chain at time $t=0$. We have set here the parameter values $\xi=0.1$ and $\eta=0.0$. (a) MSP of a left end $\left(c_{3}\right)$ Majorana mode shows collapse and revival with time, but at each revival there are fluctuations and also the peaks of revivals decrease rapidly. (b) While on the other hand when the system is quenched reversely the Majorana $\left(c_{1}\right)$ decoheres rapidly with time (with no prominet revival) and fluctuates around zero mean. 


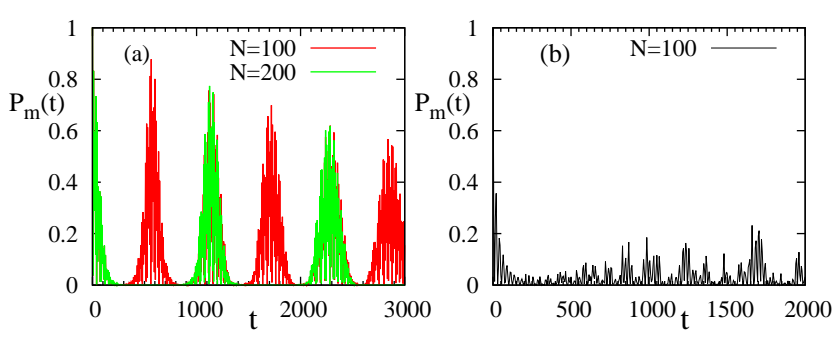

FIG. 11. Initially, the Hamiltonian $H_{2}$ is fixed at the parameter values $\xi=0.1$ and $\eta=0.0$ and then it is quenched to the critical point $(\xi=0.0$ and $\eta=0.0)$ of the Hamiltonian $H_{1}$ at $t=0$, (a) MSP of a left end $\left(c_{3}\right)$ Majorana mode shows collapse and revival with time quite similar to Fig. 10(a); the amplitude of the revival decays rapidly if the initial value of $\Delta$ is larger, i,e., the Hamiltonian $H_{2}$ is deep in the topological phase away from the QCP. (b) There is a complete loss of coherence when the system is quenched reversely.

\section{CONCLUSIONS}

In this paper, we report the possibility of survival of an edge Majorana following a sudden quenching of the p-wave superconducting chain. Analyzing the MSP, we show that when the chain quenched at the QCP separating the two topological phases (or separating one topo- logical phase and the trivial phase) the edge Majorana mode oscillates between two ends of the chain with the time period of this oscillation being proportional to the size of the spin chain. This phenomena can be attributed to nearly equispaced nature of the bulk modes and linearity of the spectrum at the QCP. However, the amplitude of oscillation eventually decreases as a function of time when the bulk modes (not equally-spaced) start contributing to the MSP. We here also consider the effect of next-nearest-neighbor hopping and supeconducting pairing in MSP. In this case the frequency of oscillation of MSP is doubled when the system is quenched to a QCP. Finally, we have looked at the instantaneous quenching between Hamiltonian $\mathrm{H}_{2}$ (with two Majoranas at each end) and $H_{1}$ (which is supposed to have one edge Majorana) when the latter is critical as well as off-critical; in both the cases, there are prominent revivals of the survival probability with the time period scaling with the system size which is a remarkable result, not apparently obvious. There is also complete detraction of coherence when one performs a quench from $H_{1}$ to $H_{2}$.

\section{Acknowledgements}

We sincerely thank Aavishkar Patel for helpful discussions and very useful comments. We also acknowledge useful discussions with Uma Divakaran, Tanay Nag and Shraddha Sharma. AR thanks IIT Kanpur for hospitality during a part of this work.
1 A. Kitaev, Physics-Uspekhi 44, 131 (2001) arXiv:cond-mat/0010440v2 (2000).

2 I. C. Fulga, F. Hassler, A. R. Akhmerov, and C. W. J. Beenakker, Phys. Rev. B 83, 155429 (2011).

3 J. D. Sau and S. Das Sarma, Nature communications 3, 964 (2012).

${ }^{4}$ R. M. Lutchyn and M. P. A. Fisher, Phys. Rev. B 84, 214528 (2011).

5 W. DeGottardi, D. Sen, and S. Vishveshwara, New J. Phys. 13, 065028 (2011).

${ }^{6}$ W. DeGottardi, D. Sen, and S. Vishveshwara, Phys. Rev. Lett. 110, 146404 (2013).

7 M. Thakurathi, A.A. Patel, D. Sen, and A. Dutta, Phys. Rev. B 88, 155133 (2013).

${ }^{8}$ W. DeGottardi, M. Thakurathi, S. Vishveshwara, and D. Sen, Phys. Rev. B 88165111 (2013).

9 J. Alicea, Rep. Prog. Phys. 75, 076501 (2012).

10 M. Z. Hasan and C.L. Kane, Rev. Mod. Phys. 82, 3045 (2010).

11 X.-L. Qi and S.-C. Zhang, Rev. Mod. Phys. 83, 1057 (2011).

12 L. Fu and C.L. Kane, Phys. Rev. Lett. 100, 096407 (2008).

13 V. Mourik et al., Science 336, 1003 (2012).

14 M.T. Deng, C.L. Yu, G.Y. Huang, M. Larsson, P. Caroff, and H.Q. Xu, Nano Lett. 12, 6414 (2012).

15 A. Das, Y. Ronen, Y. Most, Y. Oreg, M. Heiblum, and H. Shtrikman, Nat. Phys. 8, 887 (2012).
16 W. Chang, V. Manucharyan, T. Jespersen, J. Nygard, and C. Marcus, Phys. Rev. Lett. 110, 217005 (2013).

17 A. D. K. Finck, D. J. Van Harlingen, P. K. Mohseni, K. Jung, and X. Li, Phys. Rev. Lett. 110, 126406 (2013).

18 Diego Rainis, Luka Trifunovic, Jelena Klinovaja, and Daniel Loss, Phys. Rev. B, 87, 024515 (2013)

19 J. C. Budich, S. Walter and B. Tranzettel, Phys. Rev. B 85, 121405 (R) 2012.

20 M. J. Scmidt, D. Rainis and D. Loss, Phys. Rev. B 86, 085414 (2012).

21 E. Perfetto, Phys. Rev. Lett. 110, 087001 (2013).

22 M.-C. Chung, Y. -H. Jhu, P. Chen, C.-Y. Mou, and X. Wan, arXiv:1401.0433

23 A. Polkovnikov, K. Sengupta, A. Silva, and M. Vengalattore, Rev. Mod. Phys. 83, 863 (2011).

24 A. Dutta, U. Divakaran, D. Sen, B.K. Chakrabarti, T.F. Rosenbaum, and G. Aeppli, arXiv:1012.0653v2 (2010).

25 J. Dziarmaga, Adv. Phys. 59, 1063 (2010).

${ }^{26}$ W.H. Zurek, et al, Phys. Rev. Lett. 95, 105701 (2005).

27 A. Polkovnikov, Phys. Rev. B 72, 16120(R) (2005).

28 B. Damski, Phys. Rev. Lett. 95, 035701 (2005); R.W. Cherng and L.S. Levitov, Phys. Rev. A 73, 043614 (2006); V. Mukherjee, U. Divakaran, A. Dutta, and D. Sen, Phys. Rev. B 76, 174303 (2007); K. Sengupta, D. Sen, and S. Mondal, Phys.Rev.Lett.100, 077204(2008)

29 C.De Grandi, V. Gritsev, and A. Polkovnikov, Phys. Rev. B 81, 012303 (2010). 
${ }^{30}$ V. Mukherjee and A. Dutta, Phys. Rev. B 83, 214302 (2011); A.A. Patel and A. Dutta, Phys. Rev. B 86, 174306 (2012).

31 Dimitris I. Tsomokos, Alioscia Hamma, Wen Zhang, Stephan Haas, and Rosario Fazio, Phys. Rev. A 80, 060302 (2009).

32 A. Rahmani and C. Chamon, Phys. Rev. B 82, 134303 (2010).

33 G. B. Halasz and A. Hamma, Phys. Rev. Lett. 110, 170605 (2013).

34 A. Bermudez, D. Patan, L. Amico, M. A. Martin-Delgado, Phys. Rev. Lett. 102, 135702 (2009).

35 A. Bermudez, L. Amico, and M.A. Martin-Delgado, New. J. Phys. 12, 055014 (2010).

36 A.A. Patel, S. Sharma, and A. Dutta, Eur. Phys. J. B 86, 367 (2013).
37 L. Mazza, D. Rossini, M. Endres, and R. Fazio, arXiv: 1311.2615 (2013).

38 Y. Niu, S.B. Chung, C.-H. Hsu, I. Mandal, S. Raghu, and S. Chakravarty, Phys. Rev. B 85, 035110 (2012).

39 Q.-J. Tong, J.-H. An, J. Gong, H.-G. Luo, and C.H. Oh, arXiv:1211.2498 (2012).

40 E. Lieb, T. Schultz, and D. Mattis, Ann. Phys.(NY) 16, 407 (1961).

41 H. T. Quan, Z. Song, X. F. Liu, P. Zanardi, and C. P. Sun, Phys. Rev. Lett. 96, 140604 (2006).

42 S. Sharma, V. Mukherjee, and A. Dutta, Eur. Phys. J B 85, 143 (2012).

43 J. Happola and G. B. Halasz and A. Hamma, Phys. Rev. A 85, 032114 (2012). 